\title{
Traffic Routing Through Off-Line LSP Creation
}

\author{
Srecko Krile and Djuro Kuzumilovic \\ University of Dubrovnik, Department of Electrical Engineering \\ and Computing, Cira Carica 4, 20000 Dubrovnik, Croatia \\ srecko.krile@unidu.hr
}

\begin{abstract}
In the context of dynamic bandwidth allocation the QoS path provisioning for coexisted and aggregated traffic could be very important element of resource management. As we know all traffic in DiffServ/MPLS network is distributed among LSPs (Label Switching Path). Packets are classified in FEC (Forwarding Equivalence Class) and can be routed in relation to $\mathrm{CoS}$ (Class of Service). In the process of resource management we are looking for optimal LSP, taking care of concurrent flows traversing the network simultaneously. For better load-balancing purposes and congestion avoidance the LSP creation can be done off-line, possible during negotiation process. The main difference from well known routing techniques is that optimal LSP need not to be necessarily the shortest path solution as it is in the case of typical online routing (e.g. with OSPF protocol).
\end{abstract}

Keywords: on-demand resource allocation, dynamical bandwidth management, end-to-end QoS routing, SLA in DiffServ/MPLS networks.

\section{Introduction}

Using dynamic service negotiation approach for SLA (Service Level Agreement) the problem of QoS path provisioning has to be in firm correlation with bandwidth management; see [1]. Aggregated flow consisted of numerous LSPs (Label Switching Path) is coming to LSR (Label Switched Router) and has to be routed to egress router. All traffic, traversing simultaneously a DiffServ/MPLS domain, is distributed among LSPs as the resultant of the routing protocol. Some of LSPs generally traverse through the same path across the network, so they coexist on the same link with congestion probability; see [2]. In that sense the network operator has to find the optimal LSP for each aggregated flow without any possible congestion in the network; see [6]. In the context of on-demand resource allocation the load balancing technique is necessary. The main condition is: the sufficient network resources must be available for the priority traffic at any moment. In this paper such off-line routing technique is proposed. Proposed heuristic algorithm helps in bottleneck detection on the path and maintains high network resource utilization efficiency.

A brief explanation: During SLA negotiation the RM (Resource Manager) module can apply any shortest path-based routing algorithm (e.g. OSPF - Open Shortest Path First) to generate the initial LSP. At the next step all simultaneous flows caused by former contracted SLAs have to be taken in calculation, too (correlation with other LSPs); see [4]. The BB (Bandwidth Broker) will therefore check if there are enough resources to satisfy the requested $\mathrm{CoS}$ in the specified path. With such congestion control/expansion algorithm the RM can predict sufficient link resources to satisfy all 
traffic demands. If the optimal routing sequence has any congested link, that link has to be eliminated from initial LSP and procedure starts again. Alternatively, adding capacity arrangement (short-term) can produce extra cost [6]. If the calculation finds the path without congestions on the path, new SLA can be accepted and related LSP is stored in database of BB. In the moment of service invocation stored LSP can be easily distributed from BB to the MPLS network to support explicit connection [5]. In opposite the SLA cannot be accepted or it must be re-negotiated.

The proposed routing technique can be seen as the capacity expansion problem (CEP) without shortages. The mathematical model explanation is given in the section 2. Heuristic algorithm development and testing results are discussed in the section 3.

\section{CEP for Load Balancing Purpose}

The routing problem explained above can be seen as the capacity expansion problem (CEP) without shortages and it can be formulated as Minimum Cost MultiCommodity Flow Problem (MCMCF). Such problem (NP-complete) can be easily represented by multi-commodity the single (common) source multiple destination network. The diagram on fig. 1. shows the network flow representation of such problem with multiple QoS levels. Links connect $M$ core routers (LSR) on the path.

Transmission link capacities on the path are capable to serve traffic demands for $N$ different QoS levels (service classes). The $N$ levels are ranked from $i=1,2, \ldots, N$, from higher to lover quality level. Order number of the link is denoted with $m, m=1$, ...., $M+1 . r_{i, m}$ denotes traffic demand increment for additional capacity for each link on the path. $I_{i, m}$ represents the relative amount of idle capacity on the link; $I_{i 1}=0$, $I_{i, M+1}=0 . x_{i, m}$ represents the amount of used capacity and $y_{i, j, m}$ the amount of capacity for quality level $i$ on the link $m$ redirected to satisfy the lower quality level $j$.

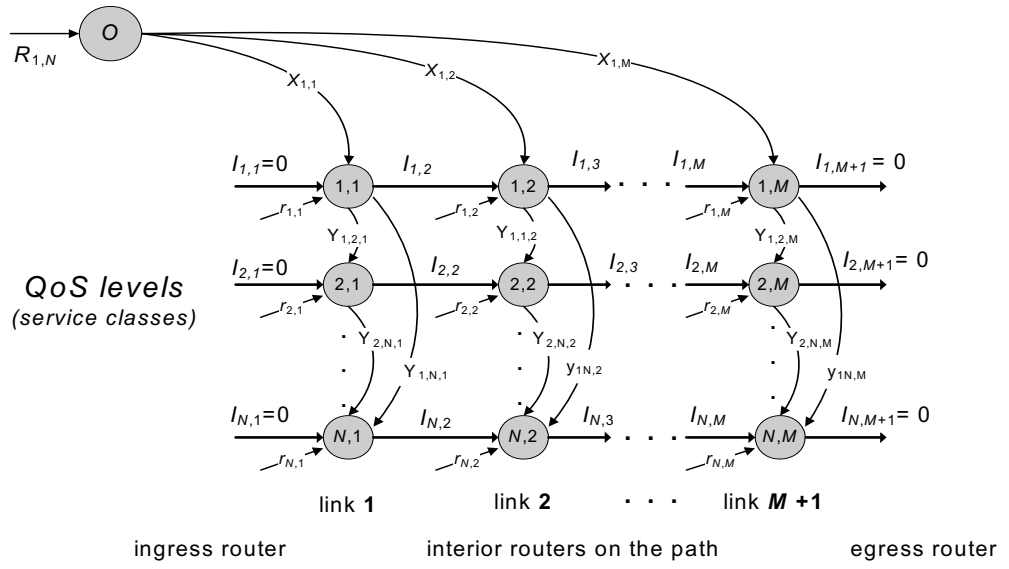

Fig. 1. The network flow representation of the CEP with application for congestion control

Let $G(A, E)$ denote a network topology, where $E$ is the set of nodes, representing link capacity states and $A$, the set of arcs representing traffic flows between routers. In this paper we talk about one-dimensional link weight vectors for $M+1$ links on the 
path $\left\{w_{i, m}, m \in A, i=1, \ldots, N\right\}$ with only one constraint, the capacity bounds, denoted with $L_{i, m}\left(L_{1, m} \mathrm{~L}_{2, m}, \ldots L_{N, m}\right)$. For a non-additive measure (e.g. bandwidth) definition of the single-constrained problem is to find a path from ingress to egress node with minimum link weight along the path. The flow situation on the link depends of expansion and conversion values $\left(x_{i, m}, y_{i, j, m}\right)$. It means that the link weight (cost) is the function of used capacity: lower amount of used capacity gives lower weight. If the link expansion cost corresponds to the amount of used capacity, the objective is to find optimal routing policy that minimizes the total cost on the path. Generalizing the concept of the capacity states we can define a capacity point:

$$
\begin{aligned}
& \alpha_{m}=\left(I_{1, m}, I_{2, m}, \ldots, I_{N, m}\right) \\
& \alpha_{1}=\alpha_{M+1}=(0,0, \ldots, 0)
\end{aligned}
$$

Each column on the flow diagram (fig. 1.) represents a capacity point, consisting of $N$ capacity state values for each. $i$-th QoS level. Each link capacity is capable to serve different service classes. The capacity class labeled with $i$ is primarily used to serve demands of that service class but it can be used to satisfy traffic of lower QoS level $j$ $(j>i)$. Formulation (2) implies that idle capacities or capacity shortages are not allowed on the link between edge and interior router.

The network optimization can be divided in two steps. In the first step we are calculating the minimal expansion weights between all pairs of capacity points. The calculation of each weight value we call it: capacity expansion sub-problem (CES).

Let $\mathrm{C}_{m}$ be the number of capacity point values at router position $m$. The total number of all sub-problem is:

$$
N_{d}=\sum_{i=1}^{M} C_{i} \cdot\left[\sum_{j=i+1}^{M+1} C_{j}\right]
$$

In the CEP we have to find many cost values $d_{u, v}\left(\alpha_{u}, \alpha_{v+1}\right)$ that emanate two capacity points, from each node $\left(u, \alpha_{u}\right)$ to node $\left(v+1, \alpha_{v+1}\right)$ for $v \geq u$. It is very important to reduce that number; see [3]. In the second step of network optimization we are looking for the shortest path in acyclic network with former calculated weights between node pairs (capacity points). It has to be noted that the optimal routing sequence for traffic (caused by new SLA) need not to be the shortest path solution.

\section{Heuristic Approach and Results}

The optimal flow theory enables separation of such extreme flows that cannot be a part of an optimal expansion solution from those which can be. So, many of expansion solutions are not acceptable. It can be shown that a feasible flow in the network (given in fig. 1.) corresponds to an extreme point solution only if it is not the part of any cycle (loop) with positive flows. This result holds for all single source networks. With such heuristic approach (denoted with BasicH) the near-optimal result is possible but significant computational savings appear, on average over than $50 \%$.

In real situation we can introduce some limitations on capacity state values, talking about different heuristic options: a) the total capacity of a capacity point can be negative but in acceptable limits (adding capacity from network provider). That 
option is denoted with $\mathrm{AH}$. b) The capacity state value inside of a capacity point can be negative, but the total sum of the link capacity is positive (denoted with RH). c) Only no-negative capacity values are allowed (denoted with $\mathrm{PH}$ ). d) Only null capacity values are allowed (TH - Trivial Heuristic option).

The heuristic algorithm with above mentioned options was tested in relation to efficiency of algorithm with exact approach. The basic heuristic approach $(\mathrm{BasicH})$ requires effort of $O\left(M^{3} N^{4} R_{i}^{2(N-1)}\right)$. For other heuristic options the complexity is significantly reduced (see fig. 2) but in all cases it is much over than $O\left(M^{3} N^{2}\right)$, that is the complexity for a trivial heuristic solution (TH). For only few test-examples all algorithm options found the best expansion sequence, providing the minimal cost no matter of which heuristic approach is used. Only in the trivial heuristic option the significant deterioration of the best result is present.

The proposed heuristic algorithm with different options can be efficiently applied in off-line routing and on-demand resource allocation, helping us in congestion avoidance and load-balancing purpose. After the starting LSP is defined (e.g. with shortest pathbased algorithm) we can check congestion probabilities on each link on the path. We can start with the algorithm of low complexity (TH or PH option). If the congestion appears we can apply more complex algorithm ( $\mathrm{RH}, \mathrm{AH}$ or $\mathrm{BasicH})$. If the congestion still exists the adding capacity is necessary or the SLA negotiation has to be continued.

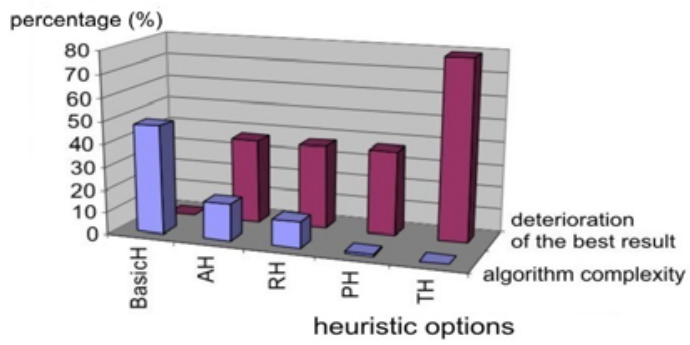

Fig. 2. Trend of the complexity changes for each heuristic option and the average value of the result deterioration

\section{References}

1. Sarangan, V., J.-C. Chen: Comparative Study of Protocols for Dynamic Service Negotiation in the Next-Generation Internet, IEEE Comm. Mag.,Vol.44, No.3 (2006) 151-156

2. Haddadou and all: Designing Scalable On-Demand Policy-Based Resource Allocation in IP Networks, IEEE Comm. Mag., Vol.44, No.3, (2006) 142-149

3. Krile, S., Kuzumilovic, D.: The Application of Bandwidth Optimization Technique in SLA Negotiation Process, Proc. of 11 th CAMAD, Trento, (2006) 115-121

4. Bhatnagar, S.; Ganguly, S.; Nath, B.: Creating Multipoint-to-Point LSPs for Traffic Engineering, IEEE Comm. Mag., Vol.43., No.1, (2005) 95-100

5. Yu Cheng and all: Virtual Network Approach to Scalable IP Service Deployment and Efficient Resource Management, IEEE Comm. Mag., Vol.43, No. 10, (2005) 76-84

6. Anjali, T., Scoglio, C., de Oliveira, J.C.: New MPLS Network Management TechniquesBased on Adaptive Learning, Trans. on Neural Networks, Vol.16, No.5, (2005) 1242 - 1255 